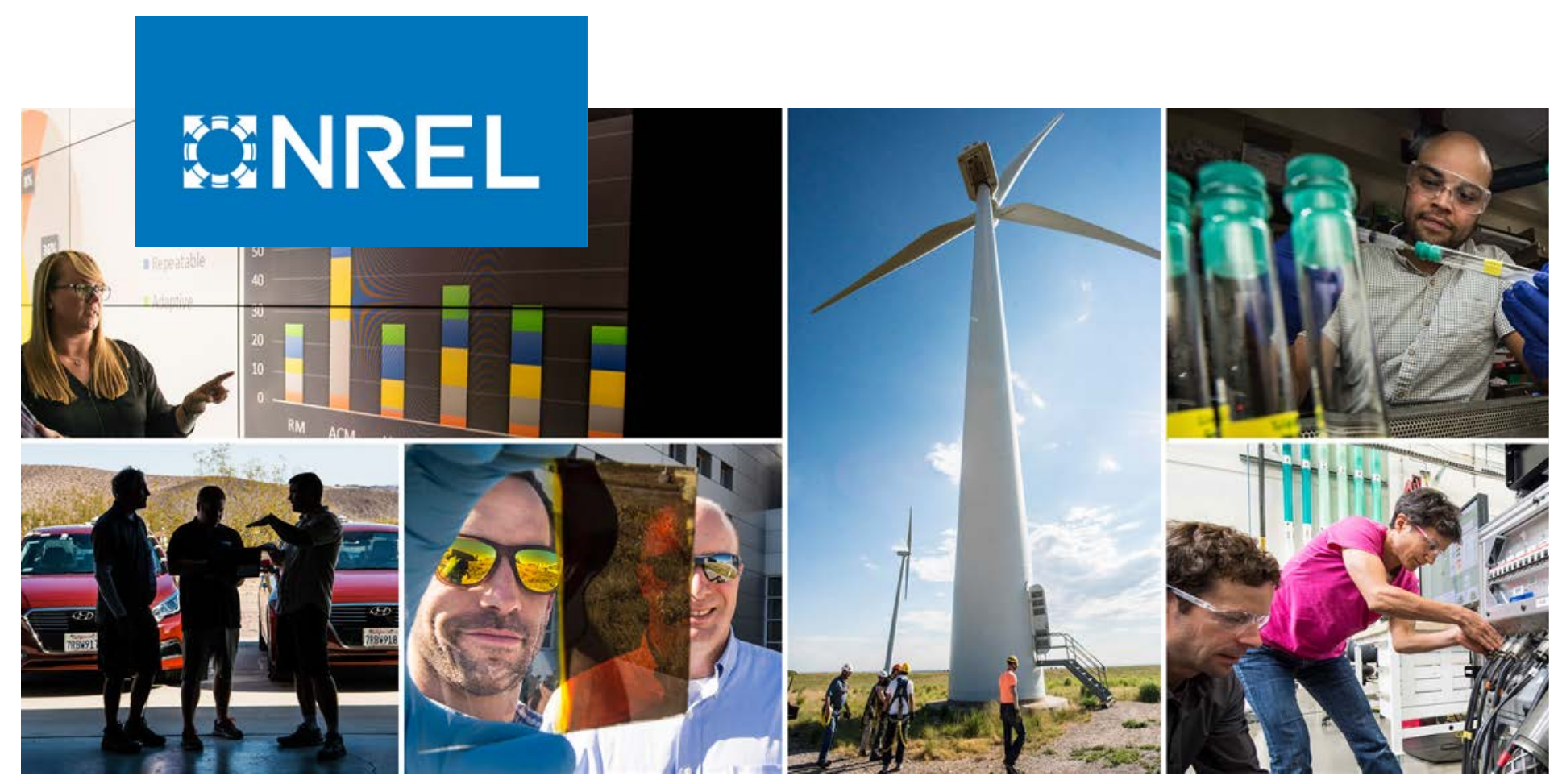

\title{
Development of Renewable Energy Data for Facilitating the Clean Energy Transition in Vietnam
}

Mohit Joshi, JoAnn Ranola, Josh Novacheck, Haiku Sky and Nick Grue

National Renewable Energy Laboratory

NREL is a national laboratory of the U.S. Department of Energy Office of Energy Efficiency \& Renewable Energy

Operated by the Alliance for Sustainable Energy, LLC

This report is available at no cost from the National Renewable Energy Laboratory (NREL) at www.nrel.gov/publications.
Technical Report

NREL/TP-5C00-80892

September 2021 


\title{
GNREL
}

\section{Development of Renewable Energy Data for Facilitating the Clean Energy Transition in Vietnam}

\author{
Mohit Joshi, JoAnn Ranola, Josh Novacheck, Haiku Sky \\ and Nick Grue
}

National Renewable Energy Laboratory

\section{Suggested Citation}

Joshi, Mohit, JoAnn Ranola, Josh Novacheck, Haiku Sky, and Nick Grue. 2021.

Development of Renewable Energy Data for Facilitating the Clean Energy Transition in Vietnam. Golden, CO: National Renewable Energy Laboratory. NREL/TP-5C00-80892. https://www.nrel.gov/docs/fy21osti/80892.pdf.

NREL is a national laboratory of the U.S. Department of Energy Office of Energy Efficiency \& Renewable Energy Operated by the Alliance for Sustainable Energy, LLC

This report is available at no cost from the National Renewable Energy Laboratory (NREL) at www.nrel.gov/publications.

Contract No. DE-AC36-08GO28308
Technical Report

NREL/TP-5C00-80892

September 2021

National Renewable Energy Laboratory 15013 Denver West Parkway Golden, CO 80401 303-275-3000 • www.nrel.gov 


\section{NOTICE}

This work was authored by the National Renewable Energy Laboratory, operated by Alliance for Sustainable Energy, LLC, for the U.S. Department of Energy (DOE) under Contract No. DE-AC36-08GO28308. Funding provided by the Children's Investment Fund Foundation through Contract No. ACT-18-00043. The views expressed herein do not necessarily represent the views of the DOE or the U.S. Government.

This report is available at no cost from the National Renewable Energy Laboratory (NREL) at www.nrel.gov/publications.

U.S. Department of Energy (DOE) reports produced after 1991 and a growing number of pre-1991 documents are available free via www.OSTI.gov.

Cover Photos by Dennis Schroeder: (clockwise, left to right) NREL 51934, NREL 45897, NREL 42160, NREL 45891, NREL 48097, NREL 46526.

NREL prints on paper that contains recycled content. 


\section{Acknowledgments}

The authors thank Ananth Chikkatur, Senior Technical Director-Energy Markets and Planning, at the U.S. Agency for International Development (USAID) Vietnam Low Emissions Energy Program II and Nathan Lee, David Palchak, and Jaquelin Cochran of the National Renewable Energy Laboratory (NREL) for their careful review and comments. We are also grateful for the editorial support from Isabel McCan and Liz Breazeale of NREL. Any errors and omissions are solely the responsibility of the authors

This work was funded by the Children's Investment Fund Foundation. 


\section{Abstract}

Vietnam is transforming its power system with an increasing share of variable renewable energy $\left(\mathrm{VRE}^{1}\right.$ ) in the generation mix and plans to achieve $27 \%$ VRE by capacity in 2030 and $42 \%$ by capacity in 2045, as indicated by the draft Eighth Power Development Plan for 2020-2045 (PDP8). Understanding the impacts of increasing VRE, as well as what may be possible beyond these targets, can be enabled by data-driven analysis where availability of data, specifically VRE data, becomes critical. NREL has worked with various stakeholders in Vietnam to create highresolution multiple-year VRE data publicly available to all. This paper reviews this new VRE data for Vietnam and examines how different stakeholders can use this data to inform VRE deployment decisions.

${ }^{1}$ VRE includes solar and wind generation for the purpose of this paper. 


\section{List of Acronyms}

CEP

LCOE

NREL

PDP8

RE

$\mathrm{reV}$

SAM

USAID

V-LEEP

VRE capacity expansion planning

levelized cost of energy

National Renewable Energy Laboratory

Eighth Power Development Plan for 2020-2045

renewable energy

Renewable Energy Potential model

System Advisor Model

U.S. Agency for International Development

Vietnam Low Emissions Energy Program

variable renewable energy 


\section{Table of Contents}

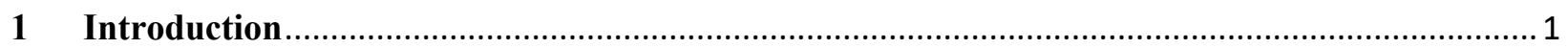

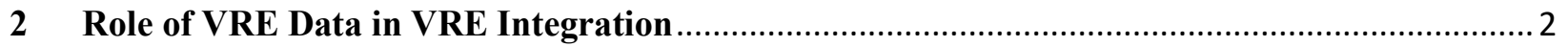

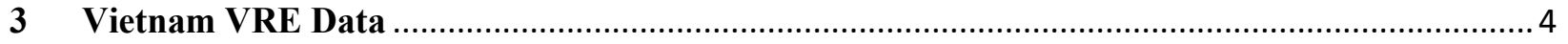

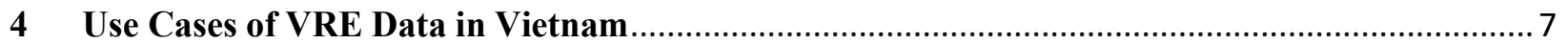

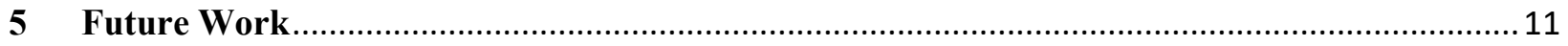

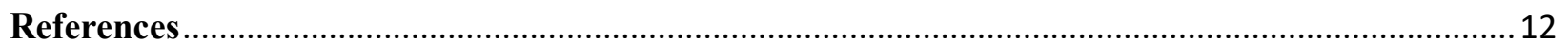




\section{List of Figures}

Figure 1. Comparison of site-specific and representative profile in MW for a sample day in each month.. 3 Figure 2. Sample technical potential (MW) of wind (left) and solar (right) in Vietnam ........................... 5

Figure 3. Capacity factor of wind (left) and solar (right) in Vietnam based on 2016 weather data............. 5

Figure 4. LCOE of solar (left) and wind (right) in Vietnam based on 2016 weather data.......................... 6

Figure 5. Average annual capacity factor of Trung Nam wind farm in Vietnam .................................... 7

Figure 6. Variability of a wind farm in Vietnam based on weather data from 2016 to 2018 of a sample day

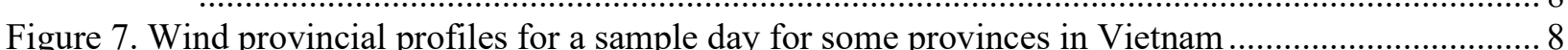

Figure 8. Regional solar profiles for a sample day for the medium and high annual capacity factor bins . 10

\section{List of Tables}

Table 1. Vietnam VRE Data Sets Available on RE Data Explorer........................................................... 4

Table 2. Solar Capacity Factor of Different Regions in Vietnam.......................................................... 9 


\section{Introduction}

The Vietnamese power sector is undergoing a transformation toward clean energy with plans to achieve $27 \%$ variable renewable energy (VRE) by capacity in 2030 and $42 \%$ by capacity in 2045. Recently, the Ministry of Industry and Trade of Vietnam released the draft Eighth Power Development Plan for 2020-2045 (PDP8). The plan proposed 18-19 GW installed wind capacity and 19-20 GW installed solar capacity by 2030 [1][2]. The existing policies such as feed-in tariff have already spurred the growth of solar in the last couple of years to a capacity of around 16-17 GW (including rooftop solar) at present [1][2]. The proposed increase in wind from $600 \mathrm{MW}$ at present to approximately $19 \mathrm{GW}$ by 2030, including offshore wind [1][2], is a significant step toward a clean energy transition. Given this considerable increase, high-resolution VRE data will help stakeholders understand the potential impacts of this growth and ensure that these targets are met. A considerable amount of coal and gas is also planned for the future in the draft PDP8. Another byproduct of publicly available high-resolution VRE data is that studies for various levels of VRE can be undertaken by key stakeholders and can drive greater understanding of the tradeoffs, possibly leading to greater ambition of VRE targets. One of the important components of any power system study or analysis is data availability and quality, and VRE data is becoming increasingly important on grids where wind and solar are providing a substantial amount of generation. With funding from the Children's Investment Fund Foundation, the National Renewable Energy Laboratory (NREL) has provided open access to multiple-year high-quality VRE data for Vietnam. This paper focuses on the VRE data developed by NREL and how this can be used by various stakeholders such as planners, system operators, and others, to conduct technical analysis and build confidence in the value and feasibility of achieving a higher share of VRE in the power system. 


\section{Role of VRE Data in VRE Integration}

Availability of data plays a crucial role in understanding the characteristics of any VRE resource and its impact on the power system. This understanding helps various stakeholders to direct their actions to support the energy transition, thereby facilitating VRE integration [3]. However, various stakeholders have different roles in VRE integration and have specific data needs. The section below defines some of these needs that this high-quality VRE data can fulfill.

1. Policymakers: Governments set VRE mandates and/or policies promoting VRE development across the country. This often requires an understanding of the country's technical potential and an estimate of levelized cost of energy (LCOE). More geographic granular information may improve the understanding further and helps in the development of region-specific policy measures.

2. Regulators: Regulators are often tasked with creating a conducive regulatory environment to facilitate VRE integration based on the policies or targets set by respective governments. Understanding LCOE and multiple-year energy generation patterns may be helpful for regulators in understanding the potential cost of energy during the tariff approval process. Also, knowledge of VRE variability is essential for framing regulations related to ancillary services and reserves.

3. Power system planners: VRE profiles, technical potential, and LCOE are fundamental to various planning studies, such as capacity expansion planning (CEP), production cost modeling, resource adequacy assessment, and resilience planning. Site-specific multipleyear data helps planners capture weather, geographic diversity, and variability, which all help improve the overall planning process. In the absence of site-specific information for planned or future buildouts, planners can also create representative provincial/regional profiles based on multiple site data, as discussed later in this paper.

4. VRE developers and investors: Site-specific capacity factors, generation patterns, and LCOE are essential considerations for VRE developers and investors while prospecting and identifying regions of interest. Availability of multiple-year VRE data helps build confidence among these stakeholders about their investments.

5. System operators: VRE profiles are also vital to various operational studies conducted by system operators. System operators rely on representative profiles in the lack of any actual historical data of existing or upcoming new VRE plants. These representative profiles are generally smooth and miss the variability associated with these plants. Figure 1 shows a comparison of site-specific and representative profiles. The blue color representative profile is smoother and misses the site-specific variability shown in the red color site-specific profile. System operators can access site-specific profiles from RE Data Explorer and the System Advisor Model (SAM) to improve their studies and realtime operation. It can also enhance the understanding of site-specific variability and help in pairing with other technologies such as energy storage. 


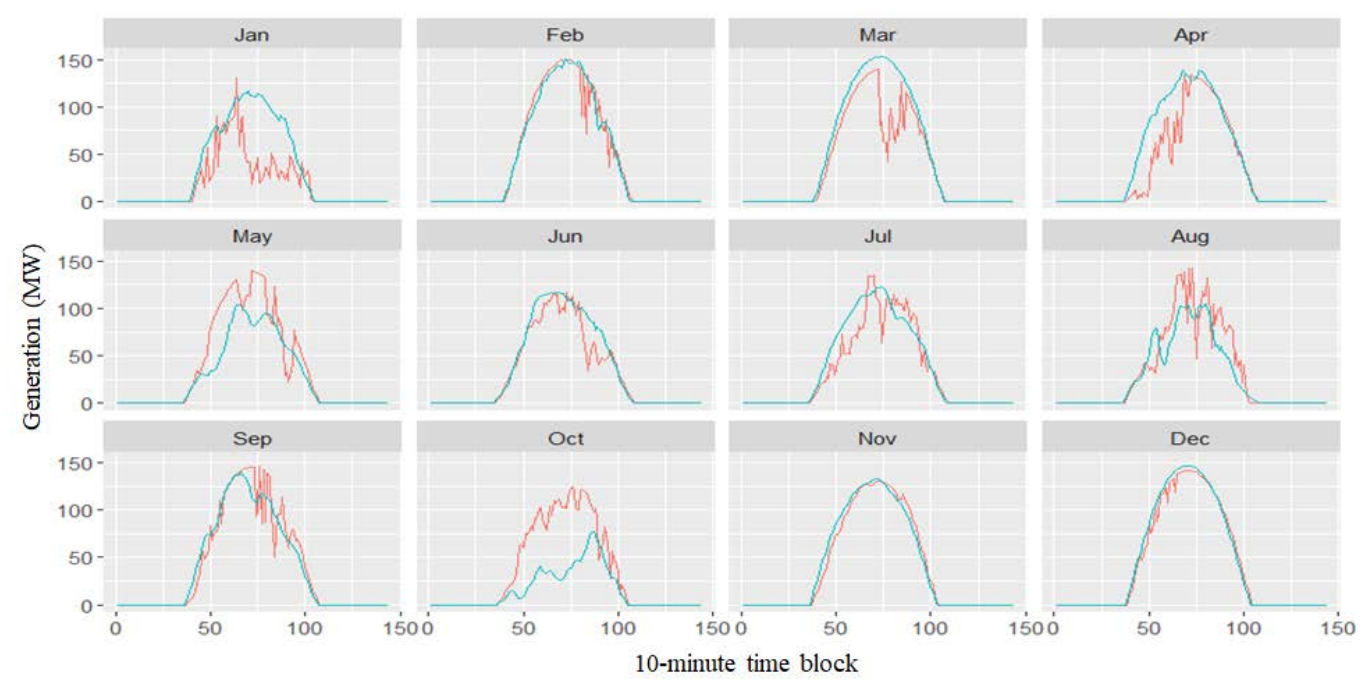

Figure 1. Comparison of site-specific and representative profile in MW for a sample day in each month

Note: Blue is a representative profile, and red is a site-specific profile.

The previous examples of how different stakeholders may use VRE data are representative, which may vary with different countries. The available data may help various stakeholders in many other ways as well. The following sections describe some Vietnam-specific use cases of VRE data. 


\section{Vietnam VRE Data}

NREL has been supporting Vietnamese stakeholders in modeling the power system impacts of achieving higher penetration of renewable generation. In partnership with the Vietnam Low Emissions Energy Program (V-LEEP) and the Hawaii Natural Energy Institute, NREL has supported the energy transition in Vietnam through capacity building and technical analyses to inform various stakeholders in PDP8 development. One of the components of this support was to develop high-quality multiple-year wind and solar resource data, as it is needed to perform various technical analyses such as CEP, production cost modeling, and other power sector models. NREL developed these data sets based on multiple-year detailed weather model and satellite data for wind and solar. Table 1 shows the details such as resolution, period, etc., of different VRE data sets developed by NREL.

Table 1. Vietnam VRE Data Sets Available on RE Data Explorer ${ }^{2}$

\begin{tabular}{|l|l|l|l|}
\hline Data Set & Year & Spatial Resolution & $\begin{array}{l}\text { Temporal } \\
\text { Resolution }\end{array}$ \\
\hline Vietnam wind data & $2016-2018$ & $2 \times 2 \mathrm{~km}$ & $1 \mathrm{hr}$ \\
\hline Vietnam solar data [4] & 2016 & $\begin{array}{l}0.05 \text { Degrees }(\sim 5.5 \times 5.5 \\
\mathrm{km})\end{array}$ & $1 \mathrm{hr}$ \\
\hline Himawari: PSM v3 & $2016-2019$ & $2 \times 2 \mathrm{~km}$ & 10 minutes \\
\hline
\end{tabular}

Multiple-year wind and solar generation time series data are publicly available on RE Data Explorer [5][6]. Users can access this data to understand various aspects of VRE sites discussed below across Vietnam. This data is either available in a raw or processed form, which users can use based on their preference. Also, users can query these data sets based on either coordinates or the selection of points or regions on the map. The available data sets include:

1. Technical attributes: Parameters such as temperature, diffuse horizontal irradiance, direct normal irradiance, wind speed at various hub heights, and wind direction are available to access on RE Data Explorer. This data can be further processed with tools like SAM and the Renewable Energy Potential (reV) model to get helpful information such as generation time series, LCOE, and technical potential [7] [8]. Users can also benefit from some level of inbuilt data processing in RE Data Explorer for these parameters.

2. Technical potential: RE Data Explorer integrates NREL's reV model to calculate the technical potential for wind and solar resources across Vietnam. The technical potential is a measure of resource potential that could be developed based on assumptions of the developable land area defined by the user. Users need to select the province(s), provide the value of their parameters (e.g., power density) for the calculation, or proceed with default values and select land exclusions. The results can be downloaded, including cumulative results (generation, land area, capacity) and land area by technical potential, or viewed on the geographic map for each location. Figure 2 shows the wind technical potential at a $100-\mathrm{m}$ hub height and solar technical potential for fixed-tilt systems across

\footnotetext{
${ }^{2}$ The Renewable Energy (RE) Data Explorer, developed by NREL, is an innovative web-based platform that allows users to visualize and analyze VRE potential.
} 
Vietnam based on 2016 data sets. The figure below is for demonstration and excludes only protected areas. Users can choose other exclusions, change technical parameters, and select different wind turbines as well.
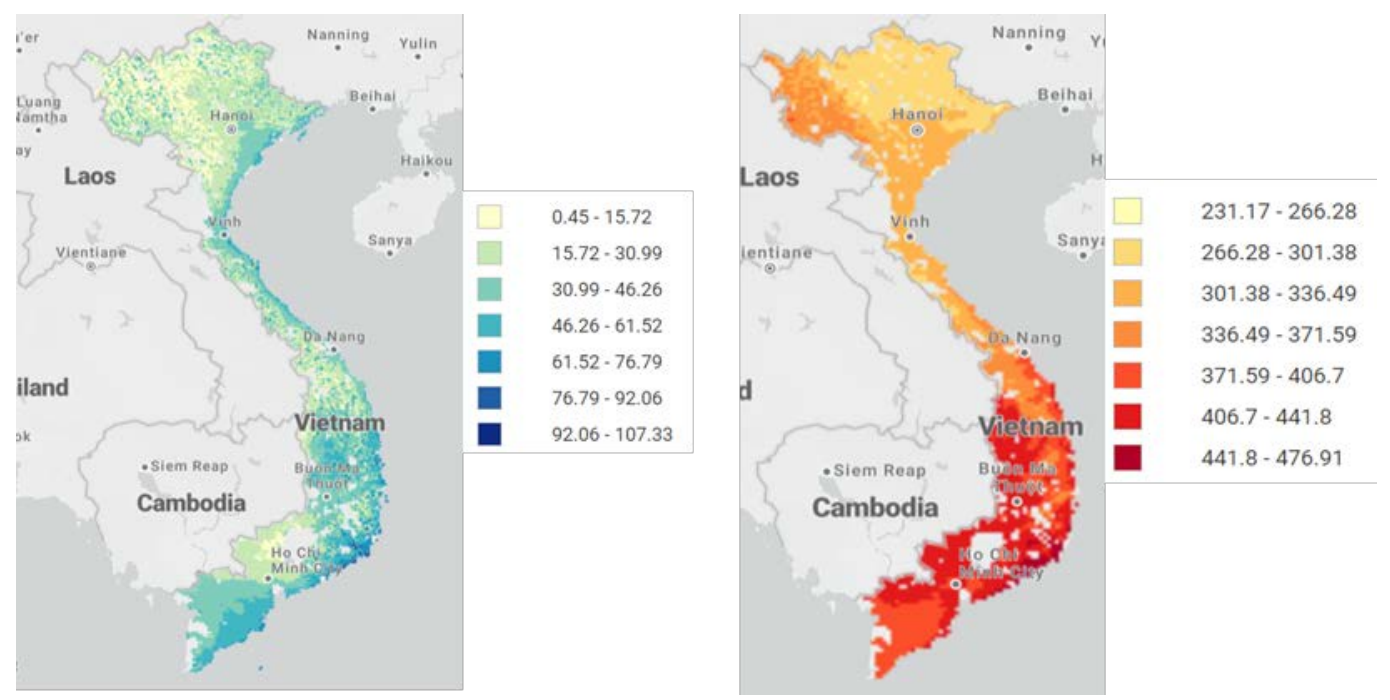

Figure 2. Sample technical potential (MW) of wind (left) and solar (right) in Vietnam

3. Capacity factor: The ratio of actual generation over a given period of time to the maximum possible generation based on total capacity is defined as capacity factor. Wind and solar generation capacity factor of every location (grids based on spatial resolution) based on 2016 data sets is readily available on RE Data Explorer. Users can download technical attribute data mentioned above and run it through SAM for other years to get capacity factors based on different years' weather data. For example, Figure 2 shows the capacity factor of wind (at 100-m hub height) and solar (fixed-tilt system) across various sites in Vietnam based on 2016 data sets.
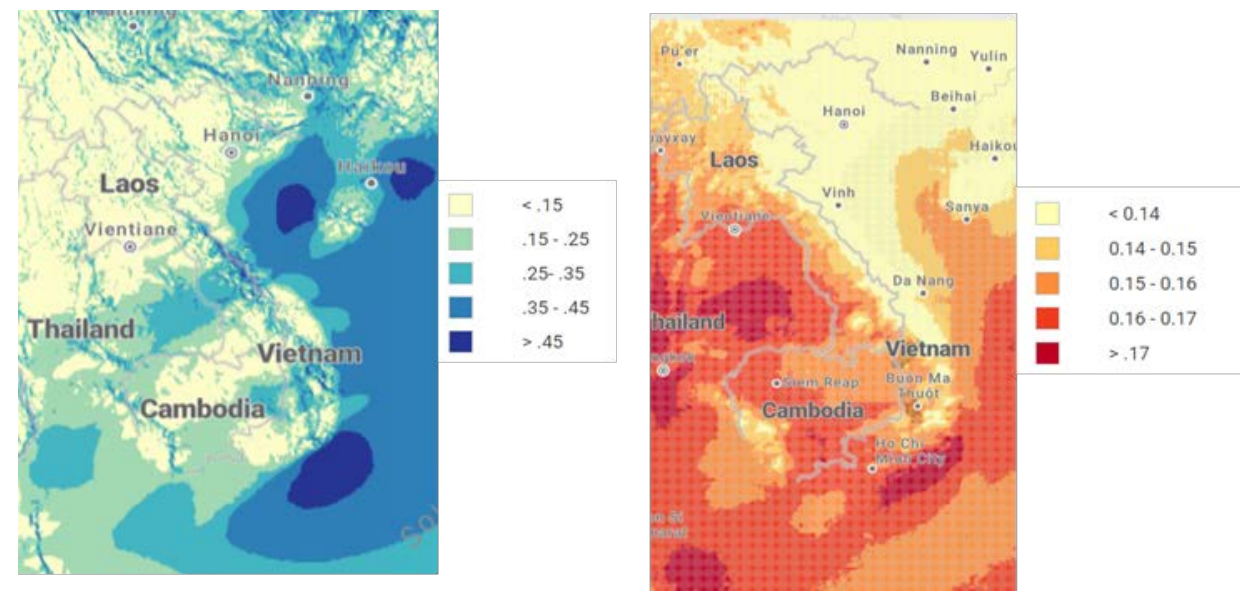

Figure 3. Capacity factor of wind (left) and solar (right) in Vietnam based on 2016 weather data

4. LCOE: RE Data Explorer also integrates the Cost of Energy Mapping Tool, which can calculate LCOE. LCOE represents the net present value of the unit cost of electricity over the lifetime of a particular electricity generation technology in dollars (USD or currency) per MWh and allows the comparison of different technologies of unequal life spans, project size, different capital cost, risk, return, and capacities. Users can calculate site- 
based LCOE that considers the energy produced at the generator's location without transmission interconnection (or access road) costs. An LCOE assessment is a part of the technical potential assessment. Figure 4 shows the wind and solar LCOE in Vietnam based on default parameters assumed for Vietnam, such as debt fraction, discount rate, lifetime, operations and maintenance costs, capital costs, exclusion of protected areas, water bodies and wetlands, forested areas, urban areas, agricultural areas, and areas with a slope greater than $5 \%$ grade.

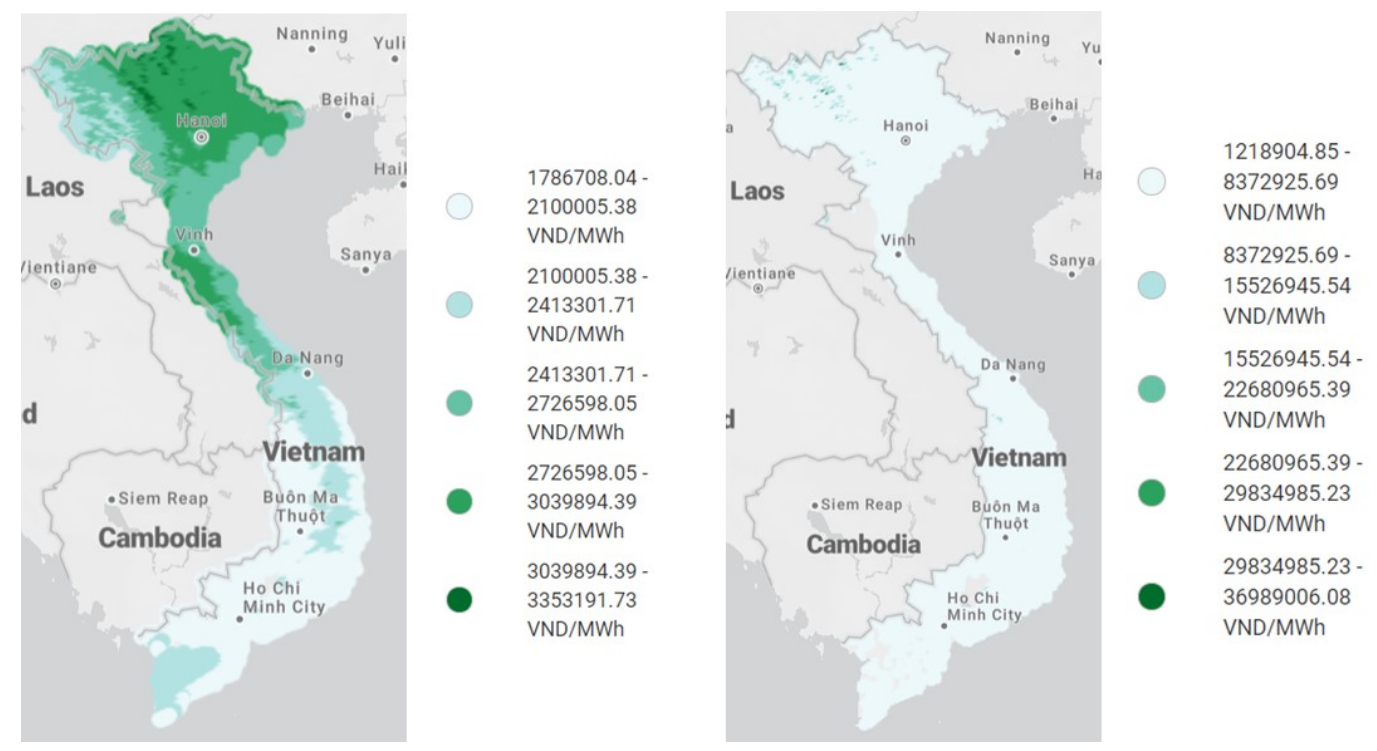

Figure 4. LCOE of solar (left) and wind (right) in Vietnam based on 2016 weather data

5. Generation output: RE Data Explorer also integrates PVWatts Calculator [9], a tool developed by NREL to estimate the energy production and cost of energy of gridconnected photovoltaic energy systems. Users can enter coordinates of any location in Vietnam and photovoltaic system configuration assumptions such as rating, array size, tilt angle, azimuth angle, and system losses to get the estimated annual, monthly, and hourly generation values. Users can also use SAM to get similar information for wind and solar based on the technical attributes for the data sets referred to previously. 


\section{Use Cases of VRE Data in Vietnam}

1. Site-specific VRE generation analysis: Multiple-year VRE data can be used to understand any VRE plant's generation characteristics (such as annual generation, monthly generation, hourly generation, variability, etc.). We use the site location of Trung Nam wind farm (Ninh Thuận province in the south-central region) to demonstrate the use of these data in understanding these characteristics. The demonstration analysis is based on hourly output in terms of capacity factor. Readers can estimate the generation (MWh/year) by multiplying the capacity factor with installed capacity (MW) and the total number of hours in one year (e.g., 8760 hours). Likewise, the output capacity (MW) can also be estimated by multiplying the capacity factor with installed capacity (MW).

Figure 5 shows the average annual capacity factor of the wind farm based on 2016-2018 weather data. It can be seen from the figure that the annual capacity factor varies with the weather year, and any decision based on a single year of data might lead to either overestimation or underestimation. Although the figure below shows an increasing trend, this may not be true for all locations as VRE generation is location- and weatherdependent. Multiple-year data can be used to calculate the exceedance probability curves [10]. Exceedance probability is defined as the amount of energy that could potentially be produced by a power plant in any given year. An exceedance probability accounts for long-term variability and weather cycles. Informed decisions can be made by considering various probabilities with annual generation.

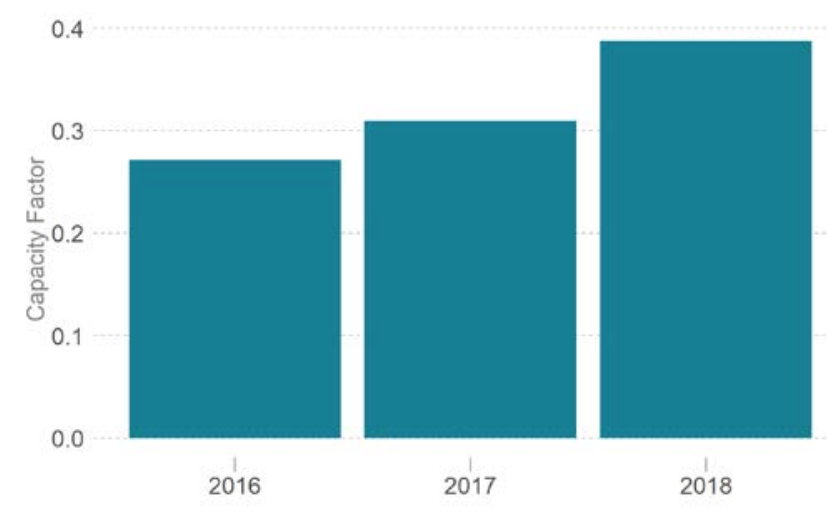

Figure 5. Average annual capacity factor of Trung Nam wind farm in Vietnam

Figure 6 shows the inter-annular variability of the same wind farm on a sample day, which refers to changes in capacity factor at the same time in different years on account of different weather conditions. The top of the gray shaded area represents the maximum generation, and the bottom represents the minimum generation on this day based on the weather data from 2016 to 2018. This information is helpful for stakeholders to examine the uncertainty related to inter-annular wind production. Rather than relying on averages, analysis can be performed on hourly data from multiple years. 


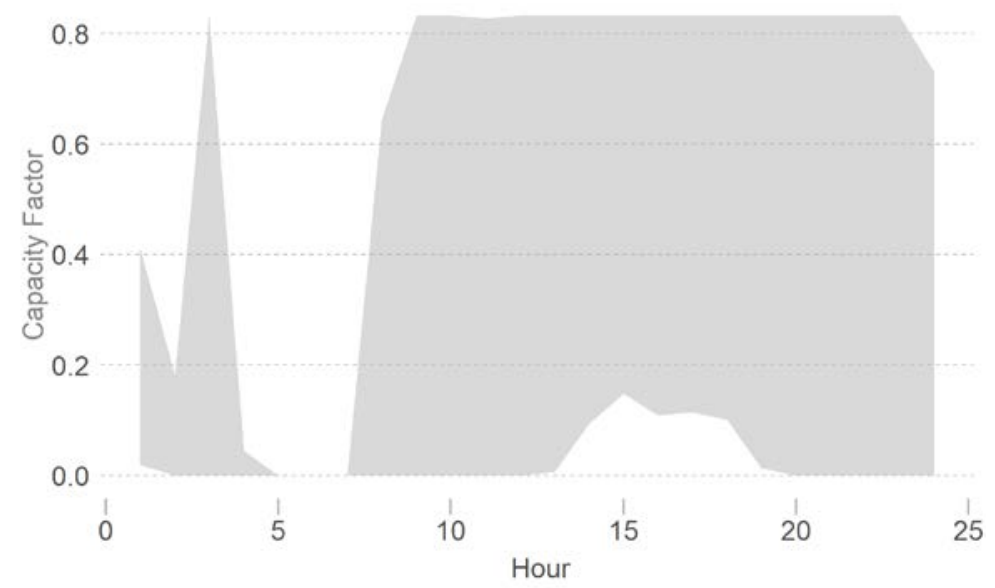

Figure 6. Inter-annual variability of a wind farm in Vietnam based on weather data from 2016 to 2018 of a sample day

2. Provincial VRE profiles for production cost modeling: As discussed in the previous section, the exact location of a future VRE plant may not be available to planners. Instead, planners may know only a wider geographic area of a future VRE plant, like province and region. In such cases, a representative profile can be used to represent the future VRE plant. This representative profile could be based on actual or modeled weather year generation data of one or multiple existing plants in that location. NREL created representative provincial profiles for the USAID study on an assessment of the revised PDP7 [11]. Solar provincial profiles were created with the assumption that top capacity factor sites would be developed first. Hence, the average of the top 25 percentile capacity factor site profiles was used to develop the solar provincial profiles. Wind provincial profiles were created assuming $1 \mathrm{GW}$ maximum possible installation in each province and each $2 \times 2 \mathrm{sq} \mathrm{km}$ site maximum buildout limit of $16 \mathrm{MW}$. Top capacity pixels (each being $16 \mathrm{MW}$ ) were selected until we got to $1 \mathrm{GW}$. The average profile of these top capacity sites is considered as a representative wind profile for that province.

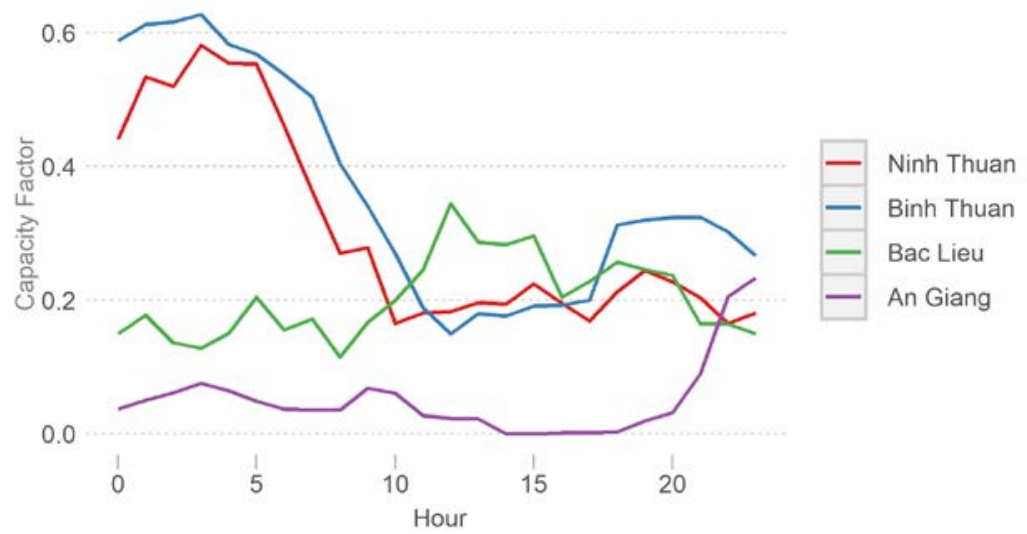

Figure 7. Wind provincial profiles for a sample day for example provinces in Vietnam 
3. Regional VRE profiles for CEP: CEP studies are undertaken to assess the quantum and type of generation capacity needed in the future. The state-of-the-art CEP tools also use VRE supply curves, build VRE based on these supply curves, and use site-specific VRE profiles. However, most of the commercially available CEP tools only need a generation profile of future VRE buildouts. This requires building representative profiles for a region. NREL created similar representative profiles for each Vietnam region for the USAID study, analyzing the impact of integrating significant VRE in Vietnam's power sector to allow CEP to utilize the high-resolution VRE data [12]. These regions are a group of Vietnam provinces defined in the USAID study referred to above. To create the profiles, NREL analyzed the capacity factor duration curve for each region to create high and medium annual capacity factor site bins. Table 2 shows the capacity factor duration curve in the tabular form. The analysis showed an evident change in the slope of the curve after 95 percentiles. This led to the inclusion of the top 5\% sites in the high bin to create a high VRE representative profile. The Medium VRE representative profile was created based on the next $20 \%$ of sites.

Table 2. Solar Capacity Factor of Different Regions in Vietnam

\begin{tabular}{|l|l|l|l|l|l|l|}
\hline Percentile & South & North & $\begin{array}{l}\text { South- } \\
\text { Central }\end{array}$ & $\begin{array}{l}\text { Center- } \\
\text { Central }\end{array}$ & $\begin{array}{l}\text { Highland- } \\
\text { Central }\end{array}$ & $\begin{array}{l}\text { North- } \\
\text { Central }\end{array}$ \\
\hline $5^{\text {th }}$ & 0.157 & 0.112 & 0.138 & 0.123 & 0.134 & 0.118 \\
\hline $15^{\text {th }}$ & 0.159 & 0.116 & 0.145 & 0.128 & 0.146 & 0.123 \\
\hline $25^{\text {th }}$ & 0.161 & 0.119 & 0.15 & 0.131 & 0.152 & 0.125 \\
\hline $35^{\text {th }}$ & 0.163 & 0.121 & 0.153 & 0.134 & 0.155 & 0.128 \\
\hline $45^{\text {th }}$ & 0.164 & 0.123 & 0.156 & 0.136 & 0.158 & 0.129 \\
\hline $55^{\text {th }}$ & 0.165 & 0.127 & 0.159 & 0.138 & 0.159 & 0.13 \\
\hline $65^{\text {th }}$ & 0.166 & 0.129 & 0.162 & 0.14 & 0.161 & 0.132 \\
\hline $75^{\text {th }}$ & 0.167 & 0.132 & 0.166 & 0.142 & 0.162 & 0.133 \\
\hline $85^{\text {th }}$ & 0.169 & 0.136 & 0.171 & 0.145 & 0.164 & 0.134 \\
\hline $95^{\text {th }}$ & 0.171 & 0.14 & 0.178 & 0.152 & 0.167 & 0.136 \\
\hline
\end{tabular}




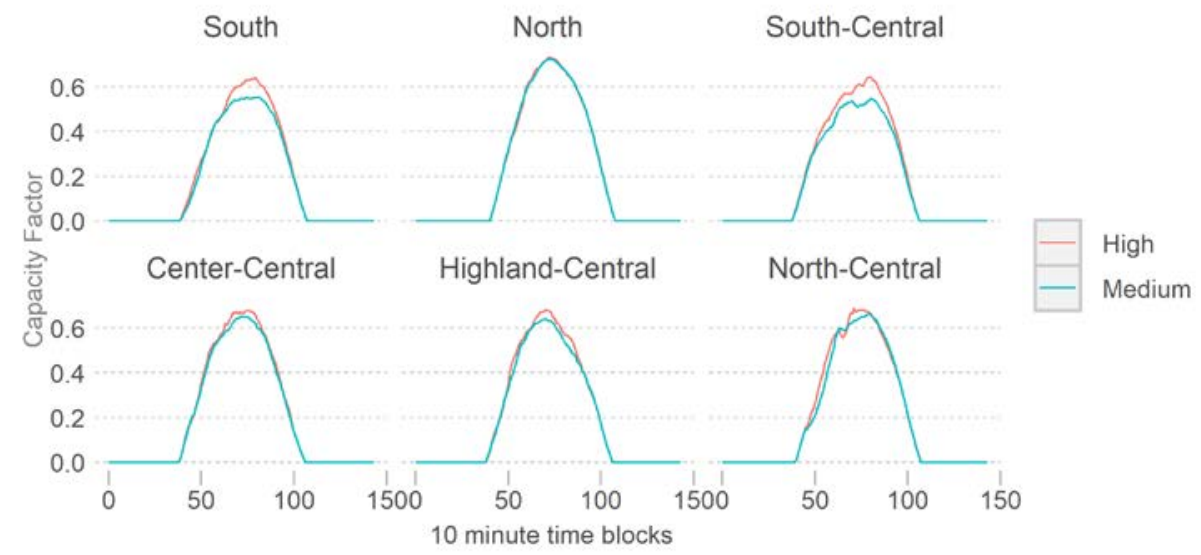

Figure 8. Regional solar profiles for a sample day for the medium and high annual capacity factor bins

4. Probabilistic Resource Analysis Suite: Resource adequacy analysis is also an important part of the planning process, which calculates metrics like expected unserved energy and loss of load expectation. Such analysis also requires VRE generation profiles. We used NREL's Probabilistic Resource Analysis Suite tool [13][14] to calculate the expected unserved energy and loss of load expectation in the USAID study, analyzing the impact of integrating significant VRE in Vietnam's power sector to allow CEP to utilize the high-resolution VRE data. 


\section{Future Work}

We will continue to support Vietnam's transition toward clean energy by providing valuable insights through more years of VRE data and producing analysis to ensure the transition to a system with high VRE penetrations is affordable, reliable, and equitable. Some of these potential analyses are:

1. VRE potential assessment: We will be developing an updated data set for VRE potentials (wind and solar) for Vietnam, using NREL's reV model. A new and improved geospatial data set will build on previously developed VRE potential analysis while more fully considering land use (e.g., urban/rural areas, protected land, difficult terrain, etc.) and power system infrastructure. The updated data set will also consider Vietnam's limited land availability, high costs, and challenging geography and its impact on grid topology. An easy-to-use interface will also be developed to allow stakeholders to readily apply the reV model to various VRE analysis, planning, and policy-related questions.

2. Resilience planning: Like other systems, Vietnam's power system also faces low probability high-impact events such as cyclones, storms, etc. These events need to be studied in detail, especially considering their impact on the system with high VRE. We will be using the multiple-year VRE data to identify historical weather events of significance, create specific weather scenarios, and simulate these scenarios using production cost modeling to serve as the framework for simulating future weather events and analyzing their impacts on operations at a national and regional level.

3. Resource adequacy analysis: Multiple-year VRE data used for resource adequacy analysis helps identify the impact of inter-annular variability on the system. We will use multiple-year VRE data to conduct resource adequacy analyses to inform various stakeholders about the expected loss of load expectation, expected unserved energy, and periods of stress. We will also extend this analysis to calculate regional capacity credits of VRE and its relationship with penetration level. This will help stakeholders improve their models with a more realistic firm capacity value of VRE. 


\section{References}

[1] EIA (U.S. Energy Information Administration). 2021. "Vietnam's latest power development plan focuses on expanding renewable sources." Accessed June 2, 2021. https://www.eia.gov/todayinenergy/detail.php?id=48176\#.

[2] Global Compliance News. 2021. "Vietnam: Key highlights of the new draft of the national power development plan (Draft PDP8)." https://globalcompliancenews.com/vietnam-keyhighlights-of-new-draft-of-national-power-development-plan-draft-pdp8-04032021-2/.

[3] Cox, Sarah L., Lopez, Anthony J., Watson, Andrea C., Grue, Nicholas W., and Leisch, Jennifer E. 2018. Renewable Energy Data, Analysis, and Decisions: A Guide for Practitioners. Golden, CO: NREL. NREL/TP-6A20-68913. https://www.nrel.gov/docs/fy18osti/68913.pdf.

[4] Huld, T., Müller, R., and Gambardella, A., 2012. "A new solar radiation database for estimating PV performance in Europe and Africa." Solar Energy 86, 1803-1815. https://www.sciencedirect.com/science/article/abs/pii/S0038092X12001119?via\%3Dihub.

[5] RE Data Explorer. "RE Explorer." Accessed June 2, 2021. https://www.re-explorer.org/redata-explorer/.

[6] Tran, J., Grue, N., and Cox, S. 2018. Renewable Energy Data Explorer User Guide. Golden, CO: NREL. NREL/TP-6A20-71532. https://www.nrel.gov/docs/fy18osti/71532.pdf

[7] NREL. "Welcome." System Advisor Model (SAM). https://sam.nrel.gov/.

[8] NREL. “The Renewable Energy Potential Model.” https://www.nrel.gov/gis/renewableenergy-potential.html.

[9] NREL. "PVWatts." https://pvwatts.nrel.gov/.

[10] Lopez, Anthony, Maclaurin, Galen, Roberts, Billy, and Rosenlieb, Evan. 2017. Capturing Inter-Annual Variability of PV Energy Production in South Asia. Golden, CO: NREL. NREL/TP-6A20-68955. https://www.nrel.gov/docs/fy17osti/68955.pdf.

[11] V-LEEP (USAID Vietnam Low Emission Energy Program). 2020. Final Report: Assessment of Revised Power Development Plan 7 by using Production Cost Model with PLEXOS. https://pdf.usaid.gov/pdf docs/PA00XGQK.pdf.

[12] V-LEEP. 2021. Impact analysis of integrating significant renewable energy in Vietnam's power sector: A PLEXOS-based analysis of long-term power development planning. https://pdf.usaid.gov/pdf docs/PA00XGQH.pdf.

[13] NREL. "PRAS.” https://nrel.github.io/PRAS/.

[14] Stephen, Gord. 2021. Probabilistic Resource Adequacy Suite (PRAS) v0.6 Model Documentation. Golden, CO: NREL. NREL/TP-5C00-79698.

https://www.nrel.gov/docs/fy21 osti/79698.pdf. 